\title{
Assessment of Exclusive Breastfeeding among Mothers Attending Immunization Clinic in Kenya: a Case Study of Kericho County
}

\author{
Article by Taratisio Ndwiga, \\ MPH student, School of Public Health, Texila American University, Kenya \\ Email: taratisiondwiga@yahoo.com
}

\begin{abstract}
Exclusive breast feeding (EBF)means that the infant receives only breast milk with no other additional foods or liquid, not even water. Breastfeeding has many health benefits for both the mother and infant.

The aim of the study was to assess factors that hide exclusive breast feeding among the mothers attending Immunization clinic in Kericho county Hospital. The target population was all the mothers with children aged 0-6 months attending immunization clinic, Kericho County Hospital. The study design was descriptive cross-sectional study. Convenience sampling was used, as quite a number of women go for immunization at the hospital. All mothers (aged 1849 years) with children 0-6 months old visiting Kericho County Hospital immunization clinic were included in the study. Fisher et al, 1998 was used to calculate the sample size, where 40 respondents were interviewed. Data was collected by use of a questionnaire. SPSS version 20.0 was used to analyze the data, which was summarized into percentages and presented in Text, figures, tables and graphs. Odd ratio was used to calculate the Respondents relationship distribution in terms of Education level versus Knowledge on EBF and Occupation versus complementary feeding.

Majority (62.5\% ) of the respondents were aged 18-27 years followed by those with 28-37 years (25\% ) while in the level of education, those with secondary were 55\%, tertiary $20 \%$ and primary were $25 \%$. For marital status, $50 \%$ were married, $32 \%$ single, $10 \%$ widowed, $5 \%$ separated and 3\% divorced. The distributions for religion, 95\% were Christians and only $5 \%$ were Muslims. In occupation self-employed were the majority $45 \%$ followed by those without employment at $35 \%$ and the employed at $20 \%$.

The sources of exclusive breastfeeding as quoted by the respondents were Health center $50 \%$, friends 25\% , CHW 12.5, Books 7.5\% and Childs grand-Mother 5\% . On the duration of EBF, those who breastfeed for 6 Months were the majority at 62.5\% , 4-5 Months at 25\% and $0-3$ Months at $12.5 \%$. On whether the EBF is possible with HIV infected mothers, $75 \%$ answered yes and not possible at 25\% and when asked how possible it was, $62.5 \%$ said through healthcare workers advice, 32.5\% through EBF only and those who did not have an idea $5 \%$.

On terms of the age of their children, respondents with 0-3 years were 60\% and 4-6 months were $40 \%$ while $42.5 \%$ had introduced other feeds such as milk, porridge, fruits, water and 57.5 had not introduced the feeds. When requested to explain their reasons to introduce others feeds, $60 \%$ said it was because of work, advice from child's grand-mother were $22.5 \%$ and those lucking enough milk were at $17.5 \%$.

The odd ratio suggested that salaried respondents are twice more likely to introduce complementary than the non-salaried respondents while those with primary education were 6 times more likely to lack knowledge on EBF than the salaried. Despite high level of knowledge on exclusive breastfeeding, challenges affecting its optimal uptake there's still need to address it in community in order to bolster its uptake. There's need to return to work by breastfeeding mothers was noted as one of barriers of exclusive breastfeeding.

The county government should make necessary legislation to create supportive work. Environment for breasting mothers who are not covered by National Maternity Policy.
\end{abstract}


South American Journal of Public Health

Special Edition May 2016

Key words: Exclusive breast feeding, Human Immunodeficiency Virus, Volunteer, Counseling and Testing, antiretroviral, Community health worker

\section{Introduction and background information}

Kericho County is found in Kenya's rift valley covering 2,479 Km2 having a population of 752, 396 people. Kericho County has 122 medical facility: 3 level 5 hospitals, 2 level 4 hospitals, 9 health centre's, 105 dispensaries and 3 VCT centers (Kericho county hospital, 2013). The county has a doctor ratio of $1: 15,000$, infant mortality rate of 35/1000 and under five mortality of $100 / 1000$. The county's poverty level is at $42.8 \%$ and an age dependency ratio of 100:87. The county's population growth rate stands at $2.4 \%$.

Exclusive breast feeding (EBF) means that the infant receives only breast milk with no other additional foods or liquid, not even water. The benefits of Exclusive breast feeding on child survival growth and development are well documented (American Academy of Pediatrics, 2012; Arun Gupta et al, 2013); breast feeding is a hygienic source of food with the right amounts of energy, proteins, fats, vitamins, and other nutrients for the infant in the first six months. Breast milk is $88 \%$ water, studies shows that healthy exclusive breast feeding of infants less than six months old do not need additional fluid, even with countries with extreme high temperatures and low humidity. Exclusive breast feeding should be given from birth up to six months and continued breast feeding, is recommended with appropriate complimentary foods until the child celebrates second birthday without water, foods and drinks. The only exceptions are rehydration salts and syrups which contains medicines (WHO, 2001 ).

Breast feeding is the most natural way to feed newborn babies, yet millions of babies are not breastfed adequately. WHO recommends exclusive breast feeding in the first six months of life and sustained breast feeding for up to two years and beyond for optimum growth, development and survival.

Exclusive breast feeding has been shown to be unsurpassed means of infant feeding that provide many health benefits to both infants and mothers. EBF may help prevent chronic diseases and conditions such as child hood obesity, type 2-diabetes and asthma. EBF for six months brings several health benefits such as decreased rates of gastrointestinal tract infections and morbidity. Breast feeding mothers may have delayed fertility, lower rate of postpartum bleeding; faster return to pre-pregnancy and resorting to formula will expose them to otitis-media, diarrhea, 3 increased susceptibility to rare diseases such as leukemia, severe lower respiratory tract infections and sudden infant death syndrome (Jessri, et al, 2013).

\section{Problem statement}

Exclusive breast feeding rates worldwide are low especially among low income less educated and minority women. Only about $38 \%$ of infants $0-6$ months old world wide are exclusively breast fed. Under nutrition is associated with $45 \%$ of children deaths. Globally in 2012, 162 million children under 5 yrs were estimated to be stunted and 51 million have low weight for height as consequence of poor feeding. Another 44 million are overweight or obese. In many developing countries only a third of breast fed infants meet the criteria of direct dietary diversity and feeding frequency that are appropriate for their age. About 800,000 children's live could be saved every year among under 5 years, if at all children 0-23 were optimally breastfed (WHO, 2014).

In Kenya based on demographic health survey, only a third of children under the age of 6 months are exclusively breastfed. The worst situation seems to be in the urban slums where most urban residents in Kenya work. Only $2 \%$ of children are exclusively breastfed for the first six months and 15\% of them stop breastfeeding by the end of one year (KNBS, 2010). According to statistics, rift valley ranks second to in neonatal mortality and child malnutrition, hence the reason for having this project base at Kericho on promotion of neonatal and maternal health with special focus on exclusive breast feeding.

\section{Justification}


Breast feeding is the only baby food that contains adequate nutritional contents, without it babies are exposed to several diseases and defects which can only be prevented naturally through breast feeding. As there various reasons why mothers don't practice Breast feeding, there was need to carry out more research. The findings of this study will help other researchers in conducting more research which will improve the uptake of exclusive breast feeding and hence the overall improvement in maternal and child health.

\section{Broad objective}

To assess factors that hide exclusive breast feeding among the mothers attending Immunization clinic in Kericho county Hospital.

\section{Knowledge}

Studies have identified insufficient support for breast feeding mothers form health care personnel obstacles to the successful uptake or continuation of EBF. Health care personnel were reported to have insufficient skills to offer remedies to deal with breast feeding problems such a painful or cracked nipples or perceived insufficient milk production. Studies have shown that a major in practicing EBF faced by mothers is the perception of insufficient breast milk production.

Inadequate information about how to stimulate milk production often leaves mothers frustrated with inadequate amount of milk, which leads them to introduce mixed feeding (Rita, 2012). From public health standpoint, it's important first to assess what factors affect women's intention to breastfeed and then analyze the obstacles that prevent them from carrying out their desires. In summery knowledge that breastfeeding is the optimal method of infant feeding is not enough to encourage women to nurse their children. Whether or not a mother breastfeeds or not is influenced by many interrelated factors, including cultural beliefs, about infant feeding practices. and duration of breastfeeding in specific populations (Goldman. A. S et al, 2007). In Kericho and other parts of Kenya, mothers have a lot of say in upbringing of their grandchildren unfortunately many don't believe a baby can be well nurtured through EBF, its prudent therefore to target not only mothers but also husband and mothers in-law in breastfeeding campaigns. There is need for a multifaceted approach to improving EBF including addressing maternal misconception related to EBF and targeting other stakeholders such as fathers and grand mother, conducting seminars in the community and religious centers and discussing issues during mother to mother support group meetings (www.jica.go.jp).

Although breastfeeding is universal in the communities, the knowledge and practice of EBF is low. There's need to institute necessary interventions aimed at early initiation of Breastfeeding and improving the knowledge and practice of EBF towards achieving the goals of MDG number 4 in the communities. Appropriate education directed at early initiation of breastfeeding, improved knowledge of EBF and use of colostrums is required to enhance EBF and duration of feeding (Moche et al, 2011 ).

Most cost effective intervention to reduce infant mortality in developing countries is promotion of EBF and appropriate complimentary feeding practices. This can be achieved by health education and public awareness programs. This would lead to achievement of the fourth MDG which is to reduce by 2/3 the mortality rate of children under five from base year 1990 to the year 2015. Hence an improvement in the feeding practice is recommended by the policy for the implementation of integrated management of neonatal illnesses. According to the study, $19.1 \%$ of neonatal deaths can be avoided with universal initiation of EBF for the first six months of life (Aatekah Owais et al, 2010).

Formal breastfeeding education is which is provided over and above the breastfeeding information given as part of standard antenatal care and which may include individual or group education sessions led by peer counselors or health professionals, home visits, lactation consultation, distribution of printed material, video demonstration and inclusion of prospective fathers in learning activities. The antenatal period affords an opportunity for 
South American Journal of Public Health

Special Edition May 2016

providing pregnant women and their partners and families with information about the benefits of breastfeeding at time when many decisions about infant feeding are being contemplated. Evidence suggests that breastfeeding education in increasing both the rate of breastfeeding initiation and duration. Randomized controlled trial demonstrated that five out or more home visits by peer counselors resulted in a significant increase in exclusive breastfeeding (Juana et al, 2013).

Although some mothers succeed in exclusive breastfeeding their infants until the age of six months and beyond, many others report introducing others foods before six months. The reason mostly given for early introduction of other foods is that the mother considers the infant to be hungry and not satisfied by breast alone. It is possible that the mothers who breastfeed their children to at least six months differ from those who do not, either in having a slower growing infant with lower energy requirements or higher breast milk energy content (Vieth, R, et al, 2007).

WHO recommends the promotion of and support of EBF in conjunction with ARV intervention as the strategy that will most likely give infants born to HIV infected mothers the greatest chance for HIV free survival? The organization recommends that when HIV infected mother's breastfeed, they should receive ARVS and exclusively breastfeed infants for six months of life, followed by prompt weaning and information of complementary foods. Breastfeeding must be interrupted based on evidence showing that the risk of HIV infection transmission through breastfeeding increases gradually as the mother breastfeed. The evidence has major implications for how mothers living with HIV might feed their infants and how health workers should counsel mothers. Together EBF and ARV intervention have the potential to significantly improve infant's chances of surviving while remaining HIV uninfected (WHO, 2010).

\section{Social Economic Factors}

Regardless of social economic status it is instrumental to enable women to exclusively breastfeed for six months and continue to breastfeed with age appropriate complementary feeds up to years and beyond. This means promoting the benefits of breastfeeding in the community level to ensure that each every mother is supported for breastfeeding mothers is particularly important in the context of the global commodity price crisis, in which young children are at increased risk of malnutrition. EBF for the six first months of life and continued breastfeeding for two years and beyond represent the best way of protect the nutritional status of young children in communities vulnerable to rising food prices (Unicef, 2007)

Mothers from low income minority groups experience a high prevalence of health problems; therefore the health benefits of EBF could positively impact this population. Unfortunately low income and minority women currently have lower probability of initiating and maintaining breastfeeding than other women. Therefore the cost effective intervention that is culturally sensitive and can be easily implemented at the community level is needed to increase EBF and duration among low income women (Foss K. \& Southwell, B. G 2006).

The reason low income women in general do not maintain EBF and instead choose formula, include the belief that breastfeeding is painful, the lack of supportive environment, embarrassment about nursing in public, concern that breastfeeding is restrictive and inconvenient, and the need to return to work (Raiser. N. S. et al, 2011 ).

When EBF is not possible due to commitment to work, a mother can manually express her milk and store it. It can be stored in a freezer storage bags and containers made specifically for breast milk. Breast milk may be kept at room temperatures up to six hours, refrigerating up to eight days or frozen for up to six to twelve months. Though research suggests that antioxidants actively degrease over time, it stills remains at higher levels than in the infant formula. Expressing breast milk can maintain a mother's milk supply when the mother and her child are apart (www.en.wikipedia.org/wiki/Breastfeeding). 
Poverty, systematic exclusion with regards to government policies and services; and lack of EBF support for a breastfeeding urban poor mother topped the list of perceived limiting factors. Extreme poverty in slums means that breastfeeding mothers lack adequate food to eat. If go to her house, she has nothing to eat. So even if you tell her to breastfeed, first that milk is not there is no food in the house. Limited livelihood opportunities mean that women have to strive to make ends meet. Newly delivered mothers have to resume work shortly after birth to fend for themselves and their families. Some women breastfeed for two months and you hear someone saying she is going back to work. You are supposed to exclusively breastfeed yet you are supposed to go to work, the way life is hard nowadays, you are forced to go to fend for yourself whether you have a baby or not. In the cash-based economic that is almost the only decent that an urban poor woman has.

Maternity leave for at least three months, a regular benefit for a non-slum residence in formal employment does not only apply. The labour system therefore excludes them from the government policy on maternity leave. Working mothers in the slums often return to work barely two weeks after delivery as they have no other forms of livelihood to rely on. Additionally, m other friendly work policies such as mothers being allowed to carry their babies to work do not apply to these mothers (Kimani-Murage E. et al, 2013).

\section{Methodology}

The study was carried out in Kericho County hospital, in Kericho County, with the target population were mothers with children aged 0-6 months attending immunization clinic, Kericho County Hospital. The Study Design was descriptive cross-sectional where Convenience sampling was used, as quite a number of women go for immunization. Women meeting the inclusion criteria were interviewed until the convenient sample size was achieved.

Fisher et al, 1998 was used to calculate the sample size, where 84 respondents were interviewed. Due to the short period, $50 \%$ of 84 were interviewed, (42)respondents rounding up to 40. Data was collected by use of a questionnaire and SPSS version 20.0 was used to analyze the data. Consent was sought from all the relevant offices within the study area through the office of CPHO and Verbal consent was sought from the respondents before the interview. Oddo ratio was employed to show the relationship of the respondent's distribution in terms of Education level versus Knowledge on EBF and Occupation versus complementary feeding.

\section{Results}

Majority (62.5\% )of the respondents were aged 18-27 years followed by those with 28-37 years $(25 \%$ )while in the level of education, those with secondary were 55\% , tertiary $20 \%$ and primary were $25 \%$. For marital status, $50 \%$ were married, $32 \%$ single, $10 \%$ widowed, $5 \%$ separated and 3\% divorced. The distributions for religion, 95\% were Christians and only $5 \%$ were Muslims. In occupation self-employed were the majority $45 \%$ followed by those without employment at $35 \%$ and the employed at $20 \%$.

On the exclusive breastfeeding knowledge, $80 \%$ were aware while $20 \%$ were not aware. The sources of exclusive breastfeeding as quoted by the respondents were health center at $50 \%$, friends at $25 \%$, CHW at $12.5 \%$, Books at $7.5 \%$ and Childs grand-mother $5 \%$. On the duration of EBF, those who breastfeed for 6 Months were the majority at $62.5 \%$, 4-5 Months at $25 \%$ and $0-3$ Months at $12.5 \%$. On whether the EBF is possible with HIV infected mothers, $75 \%$ answered yes and not possible (25\% ), among those who said it was possible, $62.5 \%$ said it was possible through healthcare workers advice, 32.5\% through EBF only and those who did not have an idea at $5 \%$. On whether mothers had enough time for breastfeeding, $62.5 \%$ responded yes while $37.5 \%$ responded No. On what mothers who work away from home can do to ensure EBF is maintained, $75.5 \%$ said the child can be taken to the work place by the care-taker for breastfeeding, $20 \%$ to leave expressed milk to the caretaker and $7.5 \%$ child to wait for the mothers return from work. 
South American Journal of Public Health

Special Edition May 2016

On terms of the age of their children, respondents with $0-3$ years were $60 \%$ and $4-6$ months were $40 \%$ while $42.5 \%$ had introduced other feeds such as milk, porridge, fruits, water and $57.5 \%$ had not introduced the feeds. When requested to explain their reasons to introduce others feeds, $60 \%$ said it was because of work commitments, advice from child's grandmother was at $22.5 \%$ and those lucking enough milk at $17.5 \%$ (Figure 2). On freedom to breastfeed in public (Figure 3), 8 Majority (70\% )felt they were free while those who did not feel free were $30 \%$. Asked to explain why they were not free to breastfeed in public, majority $(42.5 \%$ ) said it was embarrassing exposing ones breast in public, $32.5 \%$ of them said it was due to harm from the evil eye and $25 \%$ said it was due to the taboo (figure 3 ).

Table 1: Respondents distribution in terms of Education level versus Knowledge on EBF and

Occupation versus complementary feeding.

\begin{tabular}{|l|l|l|l|l|l|}
\hline \multicolumn{2}{|l|}{ Knowledge on EBF } & Yes & No & Total & Odd ratio \\
\hline Education Level & Primary & 4 & 6 & 10 & $4 / 10 * 100=40 \%$ \\
\hline & Post Primary & 28 & 2 & 30 & $2 / 3 * 100=6.67 \%$ \\
\hline & Total & 32 & 8 & 40 & RR $=40 / 6.7=5.97$ \\
\hline Any complementary feeding & Yes & No & Total & Odd ratio \\
\hline \multicolumn{7}{|l|}{} & 5 & 3 & 8 & $5 / 8 * 100=62.5 \%$ \\
\hline occupation & Salaried & 12 & 20 & 32 & $12 / 32 * 100=37.5 \%$ \\
\hline & Non-salaried & 17 & 23 & 40 & RR $=62.5 / 37.5=1.7$ \\
\hline & Total & 17 &
\end{tabular}

Those with primary education and did not know EBF were $60 \%$ while those with Post primary education and did not know EBF were $6.67 \%$. Those with primary education were 6 times more likely to lack knowledge on EBF (Table 2).

The salaried people $(62.5 \%$ )had introduced complementary feeding to their children compared to only $37.5 \%$ of the non-salaried respondents. The odd ratio suggested that salaried respondents are twice more likely to introduce complementary than the non-salaried respondents (Table 2).

\section{Source of EBF Information}

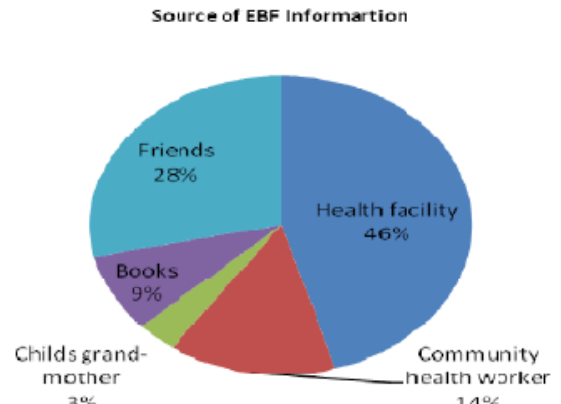
$14 \%$

Figure 1: Source of Exclusive Breastfeeding What promoted introduction of complementary feeding

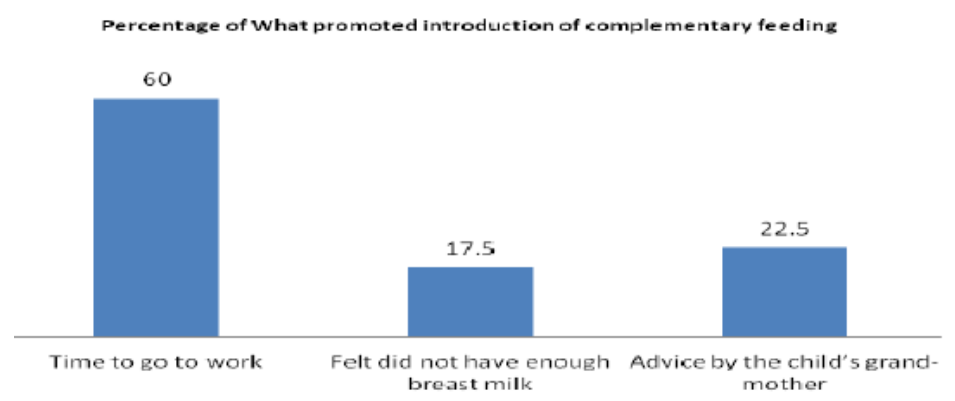


Figure 2: Percentage of what promoted introduction of complementary feeding

\section{Discussion}

Overwhelming scientific evidence supports the integral role of exclusive breastfeeding in the survival, growth and development of child as well as the wellbeing of the mother. Breast milk contains the nutritional requirements that a body needs for healthy development. it is economical , always available, clean and the right temperature., majority of the respondents were mothers between the ages of 18-27 years with (25)followed by those between 28-37 years )(10)then 38- 47 years with (25)followed by those between 28-37 years (10)then 38-47 with (5). However, this did not have a significant impact on the exclusive breastfeeding.

Regarding the level of education, majority of the respondents (55\%) attained secondary education, $25 \%$ primary level and $20 \%$ in s with tertiary education. This had a bearing in the study in that it was found out that those who had the highest level of education also had more knowledge on exclusive breastfeeding as confirmed by the odd ratio. This concurs with (Goldman A. S et al., 2007) that whether a mother breastfeeding is influenced by mainly interrelated factors. Education status of a mother is definitely one such factor.

Occupation had a critical Role to play on EBF as they showed that those with salaried employment had introduced complementary feeding. Likewise those without employment had introduced complementary feeding with perception that they did not have enough breast milk due to inadequate food. The odd ratio calculated, suggested that salaried respondents are twice more likely to introduce complementary than the non-salaried respondents. This can be attributed to working environment and distance to where they work and forces them to buy the complementary feeding feeds.

Majority of the respondents (80\%) were aware of the need to exclusively breastfeed the infants while $20 \%$ had no knowledge on what is all about. The responds indicated that $50 \%$ of those were aware got the information from the health facility, 25\% from friends, $12.5 \%$ from CHW and 7.5\% from books. This concurs with (Tomasoni L R et al, 2011 )that although breastfeeding is universal in the communities, there is need to institute necessary interventions aimed at early initiation of breastfeeding and improving the knowledge of EBF towards achieving the MDG4 in the communities.

Majority of the respondents (62.5\% )gave the right answer on the duration of the EBF from child birth while $37.7 \%$ gave the wrong answer. Most cost effective intervention to reduce infant mortalities in developing countries is promotion of EBF and this can be achieved through health education and public awareness (Shazia Memon et al., 2010).

Knowledge in whether EBF is possible with HIV infected mothers attracted mixed responses with $75 \%$ acclaiming it was possible and $25 \%$ saying contrary. Of those who claimed only 20 respondents got it right that it was possible only under instruction of a health worker and appropriate use of ARV s. WHO recommends promotion and support for EBF in conjunction with ARVs intervention as the strategy that will give infants born by HIV infected mothers the greatest chance for HIV free survival. HIV infected mothers should receive ARVs and exclusively breastfeed for six months of life followed by prompts weaning to reduce risk. Health workers should conceal these counsel these mothers. Exclusive breastfeeding with ARVs interventions have the potential to improve infant's chance of surviving while remaining HIV uninfected (WHO, 2010).

Mothers don't always have enough time for EBF as responded by $37.5 \%$ of the mothers. This is a challenge as far as exercising EBF is concern and this means that the mother and the infant are kept apart. The infant will have to miss the mother's milk for close to eight hours. On knowledge concerning what mothers committed away from home can do to ensure EBF, (29), the child is taken to the work place, (8) expressed milk should be left to the child's caretaker to feed to the infant, (3) the Childs to wait for the mothers arrival at home.

When EBF is not possible due to work commitment, a mother can manually express her milk and stores it for the child while away. Breast milk may kept at room temperature for 
upto 6 hours, refrigerated for upto 8 days or frozen for 6-12 months. This can maintain mothers supply when she and her child are apart. (www.wikipedia.com).

The study showed that $58 \%$ of the respondents had not introduced complementary feeds while $42 \%$ of them had introduced. The need to go back to work was the reason given by the majority (10)under this category. This concurred with (Kimani et al., 2013)that working mothers in the slums often return to work barely two weeks after delivery as they have no other form of livelihood to reply on. Additionally, breastfeeding mother work policies such as being allowed to carry to work do not apply to these mothers. This was followed by those who were advised by infant's grandmother (4)totally agreeing with (www.jica.go.jp)that in Kericho and other parts of rural Kenya, grandmother have a lot of say in upbringing of their grand children and many do not believe a baby can be well nurtured through EBF. Those who perceived that they did not have adequate breast milk who were (3)in number, again concurring with (www.wikipedia.com)that major barrier faced by breastfeeding mothers is the perception of insufficient breast milk production. Inadequate information about breast milk stimulation leads them to introduce complementary feeding.

Responses on the freedom to breastfeed any time whenever need arises even in public showed that $70 \%$ are free to do so. The $30 \%$ who did not feel free cited reason as, embarrassment to expose the breast in public, taboo and fear of harm from evil eyes. This concurs with (Raiser, 2011 )that the reason for women not to maintain EBF and instead choose formula includes; lack of supportive environment, embarrassment about breastfeeding in public and breastfeeding that is restrictive and inconvenient.

\section{Conclusion and recommendations}

The study found out that despite high level of knowledge on exclusive breastfeeding, challenges affecting its optimal uptake there's still need to address it in community in order to bolster its uptake. The need to return to work by breastfeeding mothers was noted as one of barriers of exclusive breastfeeding. There is also strong perception that exists among the mothers, that their breast milk is not adequate for the infants since the mother lack enough food for them to produce enough milk breast milk. Another challenge noted in the study is the influence by the family members specially grandmother of the infants who actively participate in what the infants should be fed on at a particular age. The study also found out that knowledge on breast milk as a suitable method of ensuring exclusive breastfeeding by employed and other committed mothers is low. The other challenge of concern noted in this study included fear of mothers to breastfeed in public due to taboo and fear of harm from evils eyes.

\section{Recommendations}

- The county government should make necessary legislation to create supportive work. Environment for breasting mothers who are not covered by National Maternity Policy.

- Health care staff should be trained on skills necessary for EBF implementation.

- Health education should be intensified in the health facility, schools, religion and centre and rural areas on health benefits of exclusive breastfeeding to the babies and the mothers.

- The National Government should incorporate lessons on EBF into school curriculum as school children are known to be good in disseminating messages.

- The county government should provide food subsidies to cushion the poor breastfeeding mothers.

- The county government should provide designated and secluded areas in urban areas strictly for breastfeeding only.

- The county government should conduct through social mobilization to demystify social issues affecting exclusive breastfeeding.

\section{Acknowledgements}


I warmly appreciate the help from Chief Public Health Officer in charge at The Kericho County Hospital and the entire staff. Thank you very much for your assistance.

\section{References}

[1.] Aatekah Owais, Shiyam S. Tikmani, Shazia Sultana, Umber Zaman, Imran Ahmed, Salim Allana and Anita K. M. Zaidi (2010) Incidence of pneumonia, bacteremia and invasive pnuemonoccal disease in Pakistani children. Tropical medicine \& International health. 15(90), pg 1029-1036.

[2.] American Academy of Pediatrics. Breasting feeding and the use of Human milk. Pediatrics Vol. 29 (3)2012.

[3.] Arun Gupta, J. P. Dadhich, and Shoba Suri. How Can Global Rates of Exclusive Breastfeeding for the First 6 Months be Enhanced? ICAN: Infant, Child, \& Adolescent Nutrition. Vol. x • no. x. 2013

[4.] County government of Kericho, Kericho sub-county Hospital Health records, 2015.

[5.] Denis, C. L. (2001 )Breastfeeding initiation and duration: A 1990-2000 Literature review. WHO report, 31(1).

[6.] Fisher AA, Laing JE, Strocker J E (1998) Handbook for family planning, operation research design in sampling. Population Council. 40-45.

[7.] Foss, K. A. \& Southwell, B. G (2006) Infant feeding and the media: the relationship between parents magazine content and breastfeeding. 1972-2000. International breastfeeding journal. 1(10)pg 1 $-9$

[8.] Goldman, A. S., Hopkinson, J. M., Rassin D K (2017)Benefits and risks of breastfeeding. Advances in pediatrics. Vol. 54(1 )pg. 275-304. 14

[9.] Jessri, M., Farmer, A. P., \& Olson, K. (2013). Exploring middle-eastern mother’s perceptions and experiences of breastfeeding in Canada: An ethnographic study. Maternal \& child nutrition.

[10.] Juana Willumsen (2013) breast feeding education or increased duration: biological, behavioral and contextual rationale. Dept of nutrition for health and development, WHO, Geneva. Accessed on 12th March, 2015.

[11.] Kosmala-Aderson, J., \& Wallace, L. M (2014) Breastfeeding works: The role of employers in supporting women who wish to breastfeed and work in four organizations in England. WHO journal of public health. 28(3)pg. 183-191 .

[12.] Kenya National bureau of statistics, (2010)The Kenya national health survey 2008-2009. Nairobi Kenya

[13.] Kimani-murage, E., Wekesah, F., wanjohi, M., Muriuki., Kwobutungi, C., Norris, S., \& Griffiths, P (2013) "Because most mothers are hustlers": Challenges of an urban poor 15 breastfeeding mothers in Nairobi slums. In Annals of Nutrition and metabolism. Vol. 63, pg 742-742. Basel, Switzerland.

[14.] Raiser, N. S., Laudermilk, M. J., West, J. L., Thompson, P. A \& Thomson. C. A(2011 ) recruitment of lactating women into a randomized dietary intervention: Successful strategies and factors promoting enrollment and retention. Contemporary clinical trials. 32(4).

[15.] Rita, 2012 Accessed from

www.consultancyafrica.com/index.php?option=com_content\&view=article\&id=1180:knowledge-

attitudes-and-practices-regarding-exclusive-breastfeeding-in-southern-africapart-2\&catid=61:hiv-aidsdiscussion-papers\&Itemid=268. Accessed on 12th March, 2015

[16.] Shazia Memon et. al, (2010)Assessment of infant feeding practices at a tertiary care hospital, Journal of Pakistani Medical Association, Vol. 60, (12)December, 1010-1015.

[17.] Tomasoni, L. R., Moche G., Declich, S., Pietra, V., Croce, F., Pignatelli, S., Castelli, F (2011

)Knowledge , altitude and practice regarding newborn feeding modalities in HIV - infected and HIV uninfected pregnant women in sub-Saharan Africa: A multicenter study. International health journal. 3(1)pg 56-65.

[18.] UNICEF (2007)The state of the world's children 2008. Child survival. Vol. 8, UNICEF.

[19.] Vieth, R., Bischoff-Ferreri, H., Boucher, B. J., Dawson-Hughes, B., Garland, C.F., Heaney, R. P \& Zittermann, A (2007)The urgent need to recommend an intake of vitamin Dthat is effective. The American journal of clinical medicine nutrition 85(3)pg 649-650.

[20.] www.en.wikipedia.org/wiki/Breastfeeding.Assessed 6th March, 2015.

[21.] www.jica.go.jp/kenya/english/office/topics/topics100908.html.Accessed on 7th March, 2015. 
South American Journal of Public Health

Special Edition May 2016

[22.] WHO (2010) Guidelines on HIV and infant feeding 2010: Principles and recommendations for infant feeding in the context of HIV and a summary of evidence. 